\title{
BMJ Global Health Conducting clinical research in a resource-constrained setting: lessons from a longitudinal cohort study in The Gambia
}

Abdulazeez Imam (D) , ${ }^{1}$ Oghenebrume Wariri (D) , ${ }^{1}$ Tida Dibbasey, ${ }^{1}$ Abdoulie Camara, ${ }^{1}$ Anthony Mendy, ${ }^{1}$ Assan N Sanyang, ${ }^{1}$ Masaneh Ceesay, ${ }^{1}$ Samba Jallow, ${ }^{1}$ Abdoulie E Jallow, ${ }^{1}$ Kaddijatou Bah, ${ }^{1}$ Njilan Johnson, ${ }^{1}$ Ebrima Trawally, ${ }^{1}$ Dawda Sowe, ${ }^{2}$ Alansana Darboe (D) , ${ }^{1}$ Beate Kampmann, ${ }^{1}$ Olubukola T Idoko (iD ${ }^{1}$

To cite: Imam A, Wariri 0 , Dibbasey T, et al. Conducting clinical research in a resource-constrained setting: lessons from a longitudinal cohort study in The Gambia. BMJ Global Health 2021;6:e006419. doi:10.1136/ bmjgh-2021-006419

Handling editor Seye Abimbola

$\mathrm{Al}$ and $\mathrm{OW}$ contributed equally.

Received 27 May 2021

Accepted 30 July 2021

A Check for updates

(C) Author(s) (or their employer(s)) 2021. Re-use permitted under CC BY-NC. No commercial re-use. See rights and permissions. Published by BMJ.

${ }^{1}$ Vaccines and Immunity, Medical Research Council Unit The Gambia at the London School of Hygiene and Tropical Medicine, Banjul, Gambia ${ }^{2}$ Department of Expanded Programme on Immunisation, Ministry of Health and Social Welfare, The Gambia, Banjul, Gambia

Correspondence to Dr Abdulazeez Imam; abdulimam2001@yahoo.com

\section{ABSTRACT}

Clinical research conducted to Good Clinical Practice (GCP) standards is increasingly being undertaken in resourceconstrained low-income and middle-income countries (LMICs) settings. This presents unique challenges that differ from those faced in high-income country (HIC) contexts, due to a dearth of infrastructure and unique socio-cultural contexts. Field experiences by research teams working in these LMIC contexts are thus critical to advancing knowledge on successful research conduct in these settings. The Medical Research Council Unit The Gambia at London School of Hygiene and Tropical Medicine has operated in The Gambia, a resource-constrained LMIC for over 70 years and has developed numerous research support platforms and systems. The unit was the lead clinical collaborator in a recently completed Expanded Program on Immunization Consortium (EPIC) study, involving a multicountry collaboration across five countries including the USA, Canada, Belgium, Papua New Guinea and The Gambia. The EPIC study recruited and completed follow-up of 720 newborn infants over 2 years. In this paper, we provide in-depth field experience covering challenges faced by the Gambian EPIC team in the conduct of this study. We also detail some reflections on these challenges. Our findings are relevant to the international research community as they highlight practical day-to-day challenges in conducting GCP standard clinical research in resource-constrained LMIC contexts. They also provide insights on how study processes can be adapted early during research planning to mitigate challenges.

\section{BACKGROUND}

Clinical research undertaken to internationally recognised standards is increasingly being conducted in resource-constrained low-income and middle-income countries (LMICs). This is in part related to globalisation and a skewed distribution in global disease burden. ${ }^{1}$ While high-income countries

\section{Summary box}

Globalisation has led to an increase in clinical research conducted to internationally recognised standards in many resource-constrained low-income and middle-income (LMIC) settings.

- LMIC research contexts differ significantly from those of high-income countries and field experiences of research teams operating in these areas are crucial to providing insights into the successful conduct of research in these regions.

- We present field operational challenges and how we navigated them during the conduct of a study in The Gambia.

- Our experience shows that highly supportive research platforms, flexibility, proactivity and working collaboratively with in-country governments and communities are crucial to the successful conduct of research in such settings.

(HICs) comparatively have greater research expertise, ${ }^{1}$ many diseases are more prevalent in LMICs, creating an unmet need and obligation for relevant clinical research conduct in these settings. ${ }^{2}$

Despite significant advantages, research conducted in LMIC contexts pose different challenges than would be expected in HICs. Many LMICs are characterised by poverty, fragile health systems, political instability and frequent policy changes that may impact research conduct. ${ }^{3}{ }^{4}$ Socio-cultural contexts also differ considerably from HICs, and an in-depth understanding of their dynamics is necessary for research success. ${ }^{5-8}$ Information on key processes to overcome operational challenges and local field experiences are thus critical to advancing knowledge for successful research conduct in these settings. 


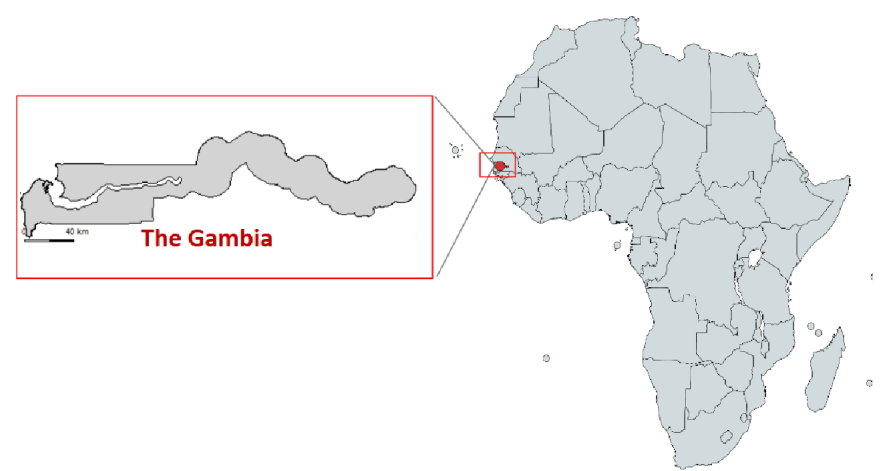

Figure 1 Map showing The Gambia in West Africa (shapefiles for our map are from https://gadm.org/data.html and https://mapchart.net/africa.html).

The Medical Research Council (MRC) Unit The Gambia at London School of Hygiene and Tropical Medicine (henceforth, MRCG at LSHTM) was the lead clinical collaborator in a recently completed Expanded Program on Immunization Consortium (EPIC) study. The EPIC is a multicountry collaboration involving partners from the USA, Canada, Belgium and Papua New Guinea. ${ }^{9}$ The study recruited and followed up 720 newborn infants over 2 years in The Gambia (figure 1); a resource-constrained LMIC. $^{9} 10$

In this paper, we highlight field operational challenges faced by the EPIC lead clinical team during our study set-up and conduct; and the processes we employed to navigate them. Our paper provides research teams planning to conduct clinical research in resource-constrained or LMIC settings with contextual and first-hand field experience of common challenges. Such information is relevant to help teams adapt their processes early on in their research planning, limit setbacks in research conduct and ensure successful and high-quality research conduct.

\section{STUDY CONTEXT \\ Research setting}

The Gambia is located in West Africa and has a population of about 2.5 million. ${ }^{11}$ It is a low-income country with $48.6 \%$ of the population living below the national poverty line. ${ }^{10}$ Like many low-income countries, The Gambia experiences infrastructural deficits. Its road networks are limited and the health system is frequently overburdened. ${ }^{12}{ }^{13}$ Healthcare is provided mainly by the government and is divided into three tiers-tertiary referral hospitals, district level facilities which provide secondary care and primary health facilities. ${ }^{12}$ We recruited our participants from two government-owned health facilities, the Banjulinding Health Centre, a primary healthcare facility, and the Kanifing General Hospital which offers secondary level care.

\section{MRCG at LSHTM}

The MRCG at LSHTM is a leading research institution in sub-Saharan Africa, which has been in existence for over

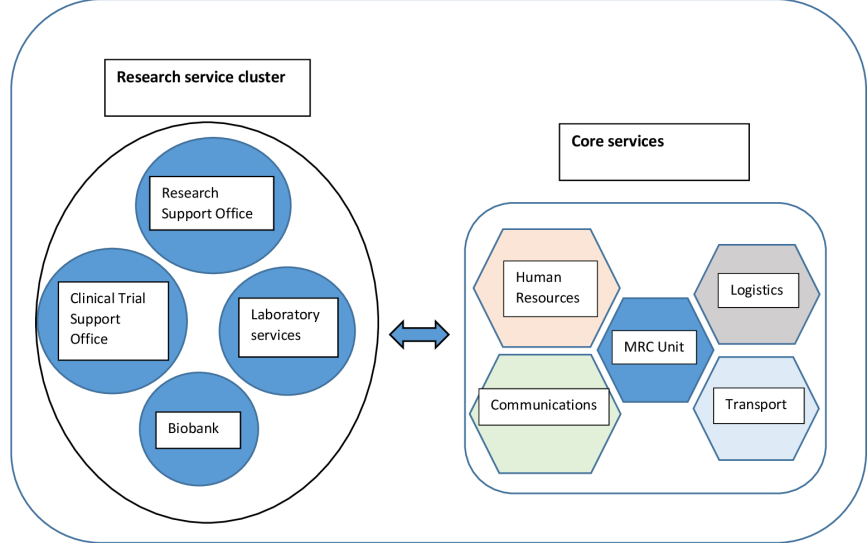

Figure 2 Diagram showing part of the research services cluster and core services at the MRC Unit.

seven decades providing scientific expertise and highquality research support platforms to facilitate successful research. These support platforms include a combination of core services and a research services cluster (figure 2). The core services include a transport department responsible for managing a vehicle and motorbike fleet; human resources, which oversees the recruitment and compensation of staff; logistics, which facilitates the receipt of supplies and transportation of goods; and communications department, which coordinates all the internal and external unit correspondences. The research services cluster, on the other hand, includes a research support office, which guides and supports research management, governance and compliance of research projects; a clinical trial support office, which ensures that clinical trials implemented at the unit are compliant with international best practices; a Biobank, which is the storage management system for all biological samples; and the laboratory services department who support research activities and patient clinical diagnosis.

\section{Brief study context}

The EPIC study recruited 720 newborn infants at birth and followed up each participant for 5 months to identify vaccine-induced molecular signatures to characterise early-life immune molecular pathways and inform future development of vaccines optimised for use in early life. ${ }^{9}$ The full research protocol for the study has previously been published elsewhere. ${ }^{9}$ The study recruited newborns in the first 24 hours of life and randomly assigned birth Expanded Program on Immunization (EPI) vaccines. This involved giving either birth dose hepatitis B (HepB) or BCG alone, or a combination of both vaccines or no vaccines and catch-up of the remaining birth vaccines (to include oral polio vaccine) on day 1,3 or 7 of life with subsequent blood draws to identify molecular signatures and determine vaccine immunogenicity.

\section{STUDY OPERATIONAL CHALLENGES}

The key challenges we experienced during the study are grouped into six themes and are summarised in table 1 . 


\begin{tabular}{|c|c|c|}
\hline Broad challenges & Specific challenges & Response \\
\hline Human resource challenge & Limited in-country specialists. & $\begin{array}{l}\text { Regional recruitment from the West- } \\
\text { African subregion. }\end{array}$ \\
\hline Vaccination policy change & $\begin{array}{l}\text { Administration of birth vaccine doses before } \\
\text { discharge to babies delivered in select government } \\
\text { hospitals. }\end{array}$ & $\begin{array}{l}\text { Speedily addressed bottleneck with } \\
\text { government authorities. }\end{array}$ \\
\hline Logistic challenges & $\begin{array}{l}\text { Limited road network. } \\
\text { Lack of a functional house address system. } \\
\text { Shipping of biosamples to northern } \\
\text { collaborators. }\end{array}$ & $\begin{array}{l}\text { Worked with the Medical Research } \\
\text { Council unit's logistic and transport } \\
\text { system. }\end{array}$ \\
\hline Participant recruitment & $\begin{array}{l}\text { Sensitised participants delivering outside the } \\
\text { study location. } \\
\text { Competing interest with another study at the } \\
\text { research site. } \\
\text { Early discharges from labour ward pressures. }\end{array}$ & $\begin{array}{l}\text { Commenced recruitment at an } \\
\text { additional facility. } \\
\text { Set up a roving team. } \\
\text { Re-organised staff rota to maximise } \\
\text { recruitments. }\end{array}$ \\
\hline Host health facility challenges & $\begin{array}{l}\text { Space constraints. } \\
\text { Neonatal sepsis outbreak. }\end{array}$ & $\begin{array}{l}\text { Built an office complex for research } \\
\text { operations. } \\
\text { Capacity building for government } \\
\text { staff on infection control and } \\
\text { newborn resuscitation. } \\
\text { Worked with and provided technical } \\
\text { assistance to host health facility to } \\
\text { tackle an outbreak. }\end{array}$ \\
\hline Rumours and misconceptions & Study-related rumours. & $\begin{array}{l}\text { Community sensitisation. } \\
\text { Prepared communication strategy to } \\
\text { address rumours. }\end{array}$ \\
\hline
\end{tabular}

ICH-GCP - International Conference on Harmonisation-Good Clinical Practice

\section{Human resource challenges}

Recruiting, following-up, generating credible data and ensuring participant safety for 720 newborn infants in a resource-constrained LMIC requires a research team skilled in providing neonatal care. At the time of conducting our study, there was a paucity of human resource skilled to provide this level of care in The Gambia.

To manage these challenges, the study recruited paediatricians from the West African subregion to support care provision for enrolled newborns and step-down newborn care training to the clinical team. We also leveraged previously established partnerships with a UK-based institution to provide additional newborn care and infection control training for research staff and government facility nurses. In keeping with standard MRCG at LSHTM procedures, all project staff also benefited from in-house Good Clinical Practice (GCP) research training. All staff received training on the study standard operating procedures and all initial research activities were performed in groups before independent operations commenced. Every station was manned by at least two study personnel as an additional quality control check allowing staff to doublecheck each step.

\section{Vaccination policy change}

Birth doses of EPI vaccine are often delayed until later in the newborn period within the Gambia. ${ }^{14}$ This is partly due to the need to prevent vaccine wastages arising from opening multidose vials out of hours when deliveries tend to occur and the need to minimise pressure on limited ward space necessitating early postpartum discharge before routine daytime immunisation clinics. It is also common for caregivers to delay vaccine birth doses past the baby's traditional naming rites occurring on the seventh day of life.

Midway through the conduct of our study, there was an unanticipated change in the Gambian vaccination strategy to address birth delays. The government piloted in-hospital administration of birth dose vaccination to newborns delivered within government hospitals before their discharge. This programme covered the main government hospitals and included one of the EPIC study sites. Its focus was to ensure timely vaccine uptake, particularly for HepB vaccine which is crucial for interrupting perinatal transmission of HepB virus (HBV) infection, as the local community prevalence of $\mathrm{HBV}$ was high at $8.8 \% .^{1415}$

The EPIC study had taken into consideration the local HBV infection burden during study design. We performed testing for maternal $\mathrm{HBV}$ infection before participant enrolment and excluded all $\mathrm{HBV}$ positive mothers, 
immediately vaccinating their newborns according to the WHO recommendation to limit the risk of transmission. The new vaccination policy meant it would be difficult to continue our study which was entirely based on randomly staggering birth dose vaccines throughout the first week of life in a population carefully screened at enrolment.

The team halted participant recruitment while securing a waiver that allowed our recruited participants to be vaccinated according to the approved project schedule. This process was greatly facilitated by existing links between the unit and the government and also our prior ethical considerations which ensured that we did not put babies at risk of infection by screening out any HepB positive mothers and families affected by tuberculosis.

\section{Logistical challenges}

Logistical challenges occur with every study and those conducted in LMICs experience unique challenges often related to a dearth of infrastructure. ${ }^{16} 17$ The limited road network in The Gambia means many feeder roads are untarred, which makes it difficult for participants to attend follow-up visits. This is particularly during the rains when whole communities could be cut-off from the township. Second, there is a lack of a functional home address system within the Gambia and it is common practice to reference a participant's residence using a local tree, or a mosque, or the house of a traditional ruler sometimes making it inefficient to trace participants, particularly in emergencies. Third, even though part of our study inclusion criteria was being resident for the subsequent 6 months within the study area, it was not uncommon for the occasional participant to travel during scheduled visits.

Additional logistical challenges included the need to conduct some of the biological assays outside the Gambia due to a lack of facility for certain select assays. Biosamples were shipped to specialised laboratories in the USA and Canada for analysis. This involved significant paperwork and applying for customs clearance, as well as providing reliable temperature control and monitoring of biosamples throughout the study and during shipment.

To address these challenges, we relied heavily on the MRCG at LSHTM transport system which has a fleet of four-wheel drives and motorcycles and has drivers who are extremely knowledgeable of the local terrain. We used this transport system to drop off and pick up newborn infants and their mothers during first-week of life study visits which was a vulnerable period for them and also for when participants were stranded due to rain or road conditions. Additionally, we had fieldworkers allocated to participants who accompanied them home alongside our project drivers following initial labour ward discharge (figure 3). This served to identify the exact participant residence and familiarise staff with immediate family members. This assigned field assistant also served as a focal contact for participant emergency communication with the team, such as in the event of illness or sudden travel. Our study protocol also included visit windows

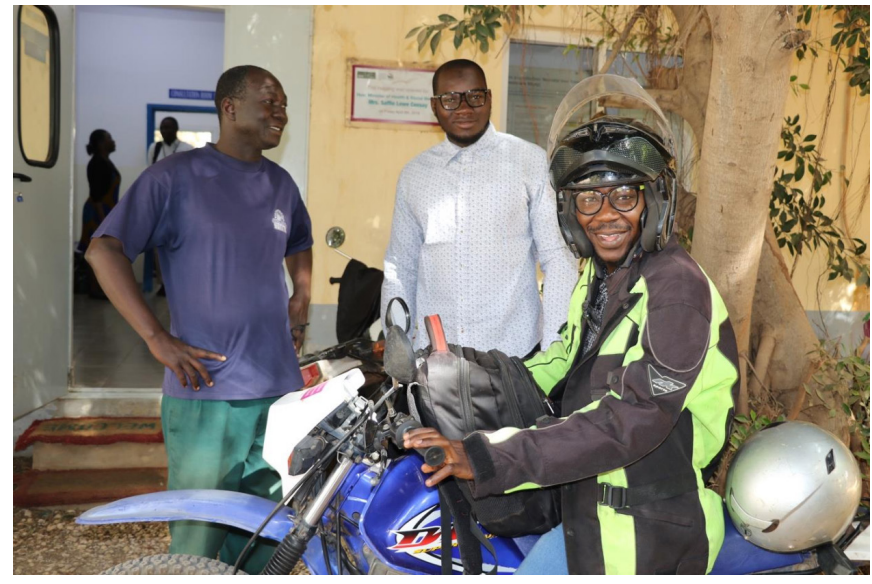

Figure 3 Dedicated field staff organising field activities; clinical activities going on in the background.

which allowed for some visit flexibility. When participant travel occurred outside these windows, we coordinated with the country EPI programme to deliver vaccines at local clinic outposts.

The team also relied heavily on the established MRCG at LSHTM Biobank for initial sample storage and the logistics department's longstanding collaborations with local shipping companies. These staff are trained to International Air Transport Association standards for storing and shipping bio-samples.

\section{Participant recruitment}

As with many clinical studies, our initial participant recruitment was relatively slow. This was partly by design to allow for smooth study protocol operationalisation and the development of team cohesion. Our operation design included ethical considerations for obtaining valid consent from women presenting in labour. We provided information to pregnant women in their second and third trimesters attending antenatal care at our study sites. This allowed women an opportunity to discuss the study with other significant decision-makers within the family, notably spouses, who are often key decision-makers in LMICs. ${ }^{18}$ Women who expressed interest in participating were encouraged to ask questions at a follow-up phone call/visit and completed the consent process when they presented in labour. However, several sensitised women did not deliver at the study site. Unanticipated changes to another study at the site meant, our participant pool became smaller than previously anticipated. Labour ward pressures for space also meant a considerable number of potential participants were discharged shortly after delivery or overnight before the team arrived for regular work hours.

As we largely anticipated these challenges, the team commenced recruitment at an additional facility and set up a roving recruitment team to recruit sensitised participants who delivered at other health facilities. Additionally, we re-organised staff rotas to maximise participant recruitment. 


\section{Host health facility challenges}

At the early stages of the study, the EPIC team experienced space constraints on the field. The labour wards and delivery rooms were frequently crowded, giving little room for research staff to operate. We also experienced an outbreak of presumed neonatal sepsis in one of our two study sites.

The MRC unit had prior knowledge of space constraints within the local health system and had sourced funds and commenced building an office complex within the health facility. Construction was completed in time to ease operations as participant numbers increased. Also, with prior knowledge of challenges relating to hospital infection control practices in LMICs, the study had involved government nurses in hands-on clean delivery room practice training and empowered our host health facilities to sustain cleaning through periodic supplementation of cleaning agents.

Following a labour ward sepsis outbreak, we worked with the local hospital authority to halt deliveries and document all neonatal sepsis cases that had occurred in the preceding weeks. We then supported the culturing of labour ward surfaces and equipment and organised the cleaning and disinfection of the facility, working together with hospital staff and community volunteers. The effect of our cleaning measures resulted in the elimination of new cases, following which we further modified our support strategy by providing manpower support for monthly health facility-led cleaning exercises. We also developed funding applications to encourage community ownership and sustainability.

\section{Rumours and misconceptions}

Rumours and misconceptions regarding research activities are typical for clinical research in varied settings. The rumours in resource-constrained settings are often linked to community distrust of research teams. ${ }^{19}$ One way to limit such rumours is patient and public involvement in all aspects of research, from the design, conduct and study reporting. However, in some instances, it might be impractical to involve the public across the full research cycle. In our case, although patients were not involved in our study design, we recognised the challenges with community information management in LMIC settings and conducted extensive community sensitisation before and during the study, and plan post-study contacts. This involved multiple meetings and engagement with members of our research communities, and their leaders locally referred to as 'Alkalos' and other key community leaders including religious, women group and village health community leaders.

Despite these efforts, rumours emanated that we were administering placebo to our study participants instead of EPI vaccines. For these rumours, we worked closely with the unit's research support office and the communications department to conduct further host community sensitisation. The departments were also prepared to engage further and provide facility tours to explain the unit's research.

\section{REFLECTIONS}

A dearth of local experts presents a major barrier facing research studies conducted in resource-constrained LMIC settings. ${ }^{20}$ In such contexts, the absence of qualified and appropriately trained study personnel could result in the collection of poor-quality data or, compromise participant safety. We regularly conduct GCP courses that ensure an up-to-date understanding of research fundamentals for new study staff and serve as refresher training for more established staff. Additionally, our recruitment of regionally-trained specialists addresses a local deficit and proffers certain advantages. These staff often have prior clinical experience practising in resource-constrained settings and provide locally relevant clinical training for their teammates given the similarities in contexts. Other capacity strengthening models described elsewhere involves employing local specialists on a part-time basis and pairing them with expatriates in a symbiotic fashion, where the local staff enhances the contextual understanding of the expatriate, who in turn supports technical know-how. ${ }^{21}$

In addition to human resource challenges, infrastructural deficits are also typical in many LMIC settings and both of these can be capital-intensive. Some research projects in LMIC have provided their own sources of water and had to generate their own electricity. ${ }^{21}$ Pre-empting these challenges during the research planning phase might mitigate them. For this reason as is common for research in this setting budget lines allowing for regional recruitment were pre-planned along with leveraging platform resources for a research office. We were able to limit other infrastructure-related costs by relying on the unit's research support platforms. An example is our reliance on the unit's transport fleet which charges a fee based on kilometres covered by research projects a cheaper alternative to acquiring project vehicles. Retaining regionally recruited staff on subsequent projects contributes to defraying costs associated with staff relocation. Where such support does not exist, non-permanent measures might serve as cheaper alternatives for the infrastructural gap. For example, previous studies at the unit have operated from modified and reconditioned shipping containers or mobile caravan units. Excellent relations with host communities have also contributed to temporary research space requirements.

Research in LMICs are also not immune to changes in external policy, as occur in any setting. During the conduct of our study, a vaccination policy change had the potential to negatively affect our recruitment. We leveraged already existing collaborations and communication channels between the unit and the Gambian government to address this. This highlights how critical it is to have the government as a stakeholder and driver of research conduct. Aside from limiting the influence of government 
policy changes on research projects, such collaboration creates greater chances of local acceptability and is a critical first step to stimulating government-led and funded research to address relevant LMIC community needs.

Managing host expectations is also important in resource-limited LMIC settings. A common narrative is that funded research projects have limitless funds and this can place such projects in challenging situations with impossible demands. It is in instances useful to consider written contractual agreements with host health facilities. As part of initial meetings with these facilities, bilaterally agreeing on the rules of engagement and also services and resources the projects can realistically provide is critical.

Finally, there is a greater chance of success for studies when research facilities possess existing research support platforms. Our study greatly benefited from the MRCG at LSHTM existing research support platforms and systems which greatly facilitated all aspects of our research.

\section{CONCLUSIONS}

Conducting clinical research in resource-constrained LMIC settings comes with unique challenges. A key to successfully conduct research to international standards requires a thorough understanding of potential challenges during planning. These are best highlighted by the host institution given the uniqueness of varied contexts. These studies also require flexibility, proactivity and working synergistically with study communities, host institutions and governments to ensure success. Additional ingredients for success are well-established research support platforms and sensitivity to the local traditions. These factors are crucial to consider to create viable research hubs that address locally relevant research questions and generate credible data acceptable to the wider research community.

Twitter Abdulazeez Imam @abdulimam2001, 0ghenebrume Wariri @drwariri and Olubukola T Idoko @bukkyidoko

\section{Acknowledgements We thank all Expanded Program on Immunization Consortium (EPIC) study staff, participants and communities in The Gambia and the wider EPIC research team for their support towards the successful completion of the study. We also acknowledge the efforts of government health staff at the labour wards and the antenatal clinics of the Kanifing General Hospital and the Banjulinding Primary Healthcare Centre in The Gambia.}

Contributors Al conceptualised the idea for this paper along with inputs from $\mathrm{OW}$. Both $\mathrm{Al}$ and $\mathrm{OW}$ co-wrote the first draft under direct supervision from OTI with inputs from BK. All coauthors critically contributed ideas to the first draft and final drafts of this paper.

Funding The EPIC study was funded primarily by the US National Institute of Health; Grant number: NIAID U19Al118608 with additional funding from the Precision Vaccines Program, Boston Children's Hospital. Core resources at the MRCG Unit at LSHTM are primarily funded via UK Research and Innovation (UKRI)

Map disclaimer The inclusion of any map (including the depiction of any boundaries therein), or of any geographical or locational reference, does not imply the expression of any opinion whatsoever on the part of BMJ concerning the legal status of any country, territory, jurisdiction or area or of its authorities. Any such expression remains solely that of the relevant source and is not endorsed by BMJ. Maps are provided without any warranty of any kind, either express or implied.

Competing interests None declared.

\section{Patient consent for publication Obtained.}

Ethics approval Ethical approvals for the Expanded Program on Immunization Consortium study was obtained from The Gambia Government/MRCG Joint Ethics Committee (Scientific Coordinating Committee number: 1513) and the Boston Children's Hospital Institutional Review Board (IRB-P00024239).

Provenance and peer review Not commissioned; externally peer reviewed.

Data availability statement There are no data in this work.

Open access This is an open access article distributed in accordance with the Creative Commons Attribution Non Commercial (CC BY-NC 4.0) license, which permits others to distribute, remix, adapt, build upon this work non-commercially, and license their derivative works on different terms, provided the original work is properly cited, appropriate credit is given, any changes made indicated, and the use is non-commercial. See: http://creativecommons.org/licenses/by-nc/4.0/.

\section{ORCID iDs}

Abdulazeez Imam http://orcid.org/0000-0001-5070-3060

Oghenebrume Wariri http://orcid.org/0000-0002-7432-8995

Alansana Darboe http://orcid.org/0000-0002-1152-8156

Olubukola T Idoko http://orcid.org/0000-0002-8802-2946

\section{REFERENCES}

1 Bowsher G, Papamichail A, El Achi N, et al. A narrative review of health research capacity strengthening in low and middle-income countries: lessons for conflict-affected areas. Global Health 2019;15:23

2 Edejer TT. North-South research partnerships: the ethics of carrying out research in developing countries. BMJ 1999;319:438-41.

3 Varmus H, Satcher D. Ethical complexities of conducting research in developing countries. N Engl J Med 1997;337:1003-5.

4 Puppalwar G, Mourya M, Kadhe G, et al. Conducting clinical trials in emerging markets of sub-Saharan Africa: review of guidelines and resources for foreign sponsors. Open Access J Clin Trials 2015;7:23-34.

5 O'Neill S, Dierickx S, Okebe J, et al. The importance of blood is infinite: conceptions of blood as life force, rumours and fear of trial participation in a Fulani village in rural Gambia. PLoS One 2016;11:e0160464

6 Akinyemi OO, Harris B, Kawonga M. 'Our culture prohibits some things': qualitative inquiry into how sociocultural context influences the scale-up of community-based injectable contraceptives in Nigeria. BMJ Open 2020;10:e035311.

7 Bannister-Tyrrell M, Gryseels C, Delamou A, et al. Blood as medicine: social meanings of blood and the success of Ebola trials. Lancet 2015;385:420.

8 Kingori P, Muchimba M, Sikateyo B, et al. 'Rumours' and clinical trials: a retrospective examination of a paediatric malnutrition study in Zambia, southern Africa. BMC Public Health 2010;10:556.

9 Idoko OT, Smolen KK, Wariri O, et al. Clinical protocol for a longitudinal cohort study employing systems biology to identify markers of vaccine immunogenicity in newborn infants in the Gambia and Papua New Guinea. Front Pediatr 2020;8:197.

10 Gambia, The | Data [Internet]. Available: https://data.worldbank.org/ country/gambia-the [Accessed 9 Jul 021].

11 Gambia Population (2021) - Worldometer [Internet]. Available: https://www.worldometers.info/world-population/gambiapopulation/ [Accessed 08 Jul 021].

12 WHO | Department of Public \& Environmental Health, Gambia [Internet]. WHO. World Health Organization. Available: https:// www.who.int/workforcealliance/members_partners/member_list/ dpehgambia/en/ [Accessed 01 Jul 021].

$13 \mathrm{GHO}$ | By category | Medical doctors [Internet]. WHO. World Health Organization. Available: https://apps.who.int/gho/data/node.main. HWFGRP 0020?lang=en [Accessed 01 Jul 021].

14 Miyahara $\bar{R}$, Jasseh M, Gomez P, et al. Barriers to timely administration of birth dose vaccines in the Gambia, West Africa. Vaccine 2016;34:3335-41.

15 Lemoine M, Shimakawa Y, Njie R, et al. Acceptability and feasibility of a screen-and-treat programme for hepatitis $B$ virus infection in the Gambia: the prevention of liver fibrosis and cancer in Africa (prolifica) study. Lancet Glob Health 2016;4:e559-67.

16 Carter RJ, Senesi RGB, Dawson P, et al. Participant retention in a randomized clinical trial in an outbreak setting: lessons from the 
Sierra Leone trial to introduce a vaccine against Ebola (strive). $J$ Infect Dis 2018;217:S65-74.

17 Idoko OT, Kochhar S, Agbenyega TE, et al. Impact, challenges, and future projections of vaccine trials in Africa. Am J Trop Med Hyg 2013;88:414-9.

18 Orobaton N, Abdulazeez J, Abegunde D, et al. Implementing at-scale, community-based distribution of misoprostol tablets to mothers in the third stage of labor for the prevention of postpartum haemorrhage in Sokoto state, Nigeria: early results and lessons learned. PLoS One 2017;12:e0170739.
19 Idoko OT, Diallo A, Sow SO, et al. Community perspectives associated with the African PsA-TT (MenAfriVac) vaccine trials. Clin Infect Dis 2015;61 Suppl 5:S416-21.

20 Alemayehu C, Mitchell G, Nikles J. Barriers for conducting clinical trials in developing countries- a systematic review. Int $J$ Equity Health 2018;17:37.

21 Carter RJ, Idriss A, Widdowson M-A, et al. Implementing a multisite clinical trial in the midst of an Ebola outbreak: lessons learned from the Sierra Leone trial to introduce a vaccine against Ebola. J Infect Dis 2018;217:S16-23. 